\title{
NEW PROOFS OF TWO $q$-ANALOGUES OF KOSHY'S FORMULA
}

\author{
EMMA YU JIN AND MARKUS E. NEBEL
}

(Communicated by Jim Haglund)

\begin{abstract}
In this paper we prove a $q$-analogue of Koshy's formula in terms of the Narayana polynomial due to Lassalle, and a $q$-analogue of Koshy's formula in terms of $q$-hypergeometric series due to Andrews, by applying the inclusion-exclusion principle on Dyck paths and on partitions. We generalize these two $q$-analogues of Koshy's formula for $q$-Catalan numbers to that for $q$-Ballot numbers. This work also answers an open question by Lassalle and two questions raised by Andrews in 2010. We conjecture that if $n$ is odd, then for $m \geq n \geq 1$, the polynomial $\left(1+q^{n}\right)\left[\begin{array}{c}m \\ n-1\end{array}\right]_{q}$ is unimodal. If $n$ is even, for any even $j \neq 0$ and $m \geq n \geq 1$, the polynomial $\left(1+q^{n}\right)[j]_{q}\left[\begin{array}{c}m \\ n-1\end{array}\right]_{q}$ is unimodal. This implies the answer to the second problem posed by Andrews.
\end{abstract}

\section{INTRODUCTION AND BACKGROUND}

Let $C_{n}=\frac{1}{n+1}\left(\begin{array}{c}2 n \\ n\end{array}\right)$ be the $n$-th Catalan number. A recursive formula for Catalan numbers is given by Koshy as follows [8]:

$$
\sum_{r=0}^{n}(-1)^{r}\left(\begin{array}{c}
n-r+1 \\
r
\end{array}\right) C_{n-r}=0 .
$$

Since this is a hypergeometric sum identity, it can be proved by Zeilberger's creative telescoping method 14. Furthermore, Koshy's formula (1.1) follows immediately from the orthogonality of a special Fibonacci polynomial due to Cigler [5, 6]. However, these approaches do not provide structural insight; to this end a combinatorial proof based on the inclusion-exclusion principle is needed. Our proof of (1.1) in the context of labeled elevated Dyck paths is exactly of this kind. Here, a Dyck path of length $2 n$ is a 2 -dimensional lattice path from $(0,0)$ to $(2 n, 0)$ with allowed steps $(1,1)(U$-step) and $(1,-1)(D$-step) that never goes below the $x$-axis. A Dyck path $p$ is an elevated Dyck path if $p=U \bar{p} D$ where $\bar{p}$ is a Dyck path. We use $D_{n}$ to denote the set of elevated Dyck paths of length $2 n+2$. Then $\left|D_{n}\right|=C_{n}$. We denote by $P$ the peak $U D$. We call $h$ an up-peak if $h=U U D$. Let $\mathcal{U P}(p)$ (resp. $\mathcal{U}(p))$ be the set of up-peaks (resp. $U$-steps) contained in a path $p$. We denote by $\left(\mathcal{M}^{s}, j, D_{n}\right)$ the set of elevated Dyck paths $p \in D_{n}$ having exactly $j$ elements from the set $\mathcal{M}(p)$ labeled by $s$. We set $a_{n, j}(\mathcal{M})=\left|\left(\mathcal{M}^{s}, j, D_{n}\right)\right|$. For the case $\mathcal{M}=\mathcal{U P}$, the number $a_{n, j}(\mathcal{U P})$ can be counted in two different ways.

Received by the editors September 4, 2013 and, in revised form, March 19, 2014 and September 10, 2014.

2010 Mathematics Subject Classification. Primary 05A19.

The work of the first author was supported by research grants from DFG (Deutsche Forschungsgemeinschaft), JI 207/1-1. 
First, there is a bijection between the set $\left((\mathcal{U P})^{s}, j, D_{n}\right)$ and the set $\left(\mathcal{U}^{s}, j, D_{n-j}\right)$. For a given path $p \in D_{n}$ that has $j$ up-peaks labeled by $s$, we can get a unique path $p^{\prime} \in D_{n-j}$ that has $j U$-steps labeled by $s$ if we remove the peak $P$ from each labeled up-peak. As a result, we have

$$
a_{n, j}(\mathcal{U P})=\left|\left((\mathcal{U P})^{s}, j, D_{n}\right)\right|=\left|\left(\mathcal{U}^{s}, j, D_{n-j}\right)\right|=\left(\begin{array}{c}
n-j+1 \\
j
\end{array}\right) C_{n-j} .
$$

Second, we set $g_{n, m}=\left|\left\{p \in D_{n}:|\mathcal{U P}(p)|=m\right\}\right|$. For a given elevated Dyck path $p \in D_{n}$ such that $|\mathcal{U} \mathcal{P}(p)|=m$, there are $\left(\begin{array}{c}m \\ j\end{array}\right)$ ways to label $j$ up-peaks by $s$, which leads to $a_{n, j}(\mathcal{U P})=\sum_{m \geq j}\left(\begin{array}{c}m \\ j\end{array}\right) g_{n, m}$. In combination with (1.2), we obtain

$$
\sum_{m \geq j}\left(\begin{array}{c}
m \\
j
\end{array}\right) g_{n, m}=a_{n, j}(\mathcal{U P})=\left(\begin{array}{c}
n-j+1 \\
j
\end{array}\right) C_{n-j} .
$$

Let $G_{n}(q)$ be the ordinary generating function of $g_{n, m}$, i.e., $\sum_{m \geq 1} g_{n, m} q^{m}=G_{n}(q)$. Then from (1.3), we can derive

$$
G_{n}(q)=\sum_{j \geq 0} \frac{G_{n}^{(j)}(1)}{j !}(q-1)^{j}=\sum_{j \geq 0}\left(\begin{array}{c}
n-j+1 \\
j
\end{array}\right) C_{n-j}(q-1)^{j},
$$

where $G_{n}^{(j)}(1)=\left.\frac{\partial^{j} G_{n}(q)}{\partial q^{j}}\right|_{q=1}$. By setting $q=0$ on both sides of (1.4), Koshy's formula (1.1) follows. Our proof uses the generating function approach to illustrate the inclusion-exclusion principle; see Chapter 2.3 of $[12$ and Chapter 4.2 of 13 . Here we will use this approach to prove (1.6) and (1.7). We can also prove the sieving identity by a direct application of the inclusion-exclusion method. To make this point clear, we will prove (2.3) in both ways in Section 2, Next we give two $q$-analogues of Koshy's formula due to Lassalle and Andrews [3, 10]. The Narayana number $N_{n, k}$ and the Narayana polynomial $N_{n}(q)$ are defined by

$$
N_{n, k}=\frac{1}{n}\left(\begin{array}{c}
n \\
k-1
\end{array}\right)\left(\begin{array}{l}
n \\
k
\end{array}\right), \quad N_{n}(q)=\sum_{k=1}^{n} N_{n, k} q^{k-1},
$$

where $N_{n, k}$ counts the number of Dyck paths of length $2 n$ that have $k$ peaks $P$. By utilizing a $\lambda$-identity of complete functions, Lassalle [10] proved for $n \geq 1$,

$$
N_{n}(q)=(1-q)^{n-1}+q \sum_{k=1}^{n-1} N_{n-k}(q) \sum_{m=0}^{k-1}(-1)^{m}\left(\begin{array}{c}
k-1 \\
m
\end{array}\right)\left(\begin{array}{c}
n-m \\
k
\end{array}\right)(1-q)^{k-m-1} .
$$

Furthermore, Andrews gave another $q$-analogue of Koshy's formula in terms of $q$ hypergeometric series. Here we adopt the standard notations of $q$-series, i.e.,

$$
\begin{aligned}
{[n]_{q} } & =1+q+\cdots+q^{n-1},[n]_{q} !=[1]_{q}[2]_{q} \cdots[n]_{q}, \\
(x ; q)_{n} & =(1-x)(1-q x) \cdots\left(1-q^{n-1} x\right), \\
{\left[\begin{array}{c}
m \\
n
\end{array}\right]_{q} } & =\frac{[m]_{q} !}{[n]_{q} ![m-n]_{q} !}=\frac{(q ; q)_{m}}{(q ; q)_{n}(q ; q)_{m-n}}, \\
C_{n}(q) & =\frac{1}{[n+1]_{q}}\left[\begin{array}{c}
2 n \\
n
\end{array}\right]_{q} .
\end{aligned}
$$


Then the before-mentioned $q$-analogue of Koshy's formula is given by

$$
\begin{aligned}
C_{n}(q) & =\sum_{r=1}^{n}(-1)^{r-1} T_{r}(n, q), \\
T_{r}(n, q) & =q^{r^{2}-r} \frac{\left(-q^{n-r+1} ; q\right)_{r}}{(-q ; q)_{r}} \cdot\left[\begin{array}{c}
n-r+1 \\
r
\end{array}\right]_{q} \cdot C_{n-r}(q) .
\end{aligned}
$$

Andrews 3] raised open questions about $T_{r}(n, q)$, which are:

(1) Is $T_{r}(n, q)$ a polynomial in $q$ ?

(2) Does $T_{r}(n, q)$ only have nonnegative coefficients of $q$ if $n \geq 2 r$ ?

(3) Does $T_{r-1}(2 r-1,-q)$ only have nonnegative coefficients?

(4) What is the partition-theoretic combinatorial interpretation of $T_{r}(n, q)$ for $n \geq 2 r$, what is that of $T_{r-1}(2 r-1,-q)$, and what is the sieving process on the partitions to eliminate all the non-Catalan partitions?

In Section 4 we completely answer (1) and (3), and show (2) for even $n$. Furthermore, in Sections 2 and 5 we prove (1.6) and (1.7) by the inclusion-exclusion method. In Sections 3 and 6 we generalize (1.6) and (1.7) for $N_{n}(q)$ and $C_{n}(q)$ to that for Ballot numbers $B_{n, r}$ and $q$-Ballot numbers $B_{r}(n, q)$. Finally we conjecture that if $n$ is odd, then for $m \geq n \geq 1$, the polynomial $\left(1+q^{n}\right)\left[\begin{array}{c}m \\ n-1\end{array}\right]_{q}$ is unimodal. If $n$ is even, for any even $j \neq 0$ and $m \geq n \geq 1$, the polynomial $\left(1+q^{n}\right)[j]_{q}\left[\begin{array}{c}m \\ n-1\end{array}\right]_{q}$ is unimodal. This would answer question (2) from above for odd $n$.

\section{Proof of (1.6)}

Before we prove (1.6), we give some main ingredients needed in the proof.

First we introduce the notions of a tower and a colored tower for an elevated Dyck path. We will choose to label the colored towers by $s$ in order to apply the inclusion-exclusion method. Recall that $D_{n}$ is the set of elevated Dyck paths of length $2 n+2$. For any $p \in D_{n}$, we will use $U^{i} D^{j} \subseteq p$ to express the fact that $p$ contains $i$ consecutive $U$-steps that are followed by $j$ consecutive $D$-steps. We call $t$ a tower of height $i$ contained in an elevated Dyck path $p$, if $t=U^{i} D^{i} \subseteq p$ and $U t D \nsubseteq p$. For any elevated Dyck path $p \in D_{n}$, let $\mathcal{T}^{*}(p)$ be the set of towers contained in $p$. We will color the towers in $\mathcal{T}^{*}(p)$ in the following way for any $p \in D_{n}$ :

Step 1. Color all the towers $t \subseteq p$ that immediately follow a $U$-step.

Step 2. Color all the towers $t \subseteq p$ that immediately follow an uncolored tower $t^{\prime} \subseteq p$ after Step 1.

We use $\mathcal{T}(p) \subseteq \mathcal{T}^{*}(p)$ to denote the set of towers contained in a path $p$ which are colored according to Steps 1 and 2. We call every $t$ in the set $\mathcal{T}(p)$ a colored tower. We set $\mathcal{T}^{c}(p)=\mathcal{T}^{*}(p)-\mathcal{T}(p)$ and let $\mathcal{T}_{2}(p) \subseteq \mathcal{T}(p)$ (resp. $\mathcal{T}_{1}(p) \subseteq \mathcal{T}(p)$ ) be the set of colored towers of height $\geq 2$ (resp. height $=1$ ). As an example, consider the path $p=U^{3} D^{2} U D U D^{2} \in D_{4}$. The colored towers in the set $\mathcal{T}(p)$ are depicted using double lines in the rightmost elevated Dyck path of Figure 1. After Step 1, the first tower in the left-to-right order is colored. The second tower remains uncolored after Step 1, and therefore after Step 2, the third tower is colored.

Second we will use some one-to-one correspondences between two sets of labeled elevated Dyck paths. If there is a bijection between two sets $A$ and $B$, we write 

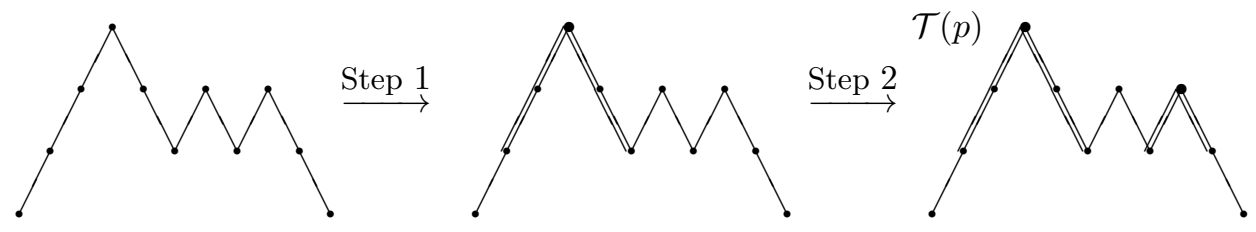

Figure 1

$A \simeq B$. Recall that $\left(\mathcal{M}^{s}, j, D_{n}\right)$ is the set of elevated Dyck paths $p \in D_{n}$ having exactly $j$ elements from the set $\mathcal{M}(p)$ labeled by $s$. We consider the set of elevated Dyck paths $p \in D_{n}$ that have $m$ colored towers labeled by $s$, which is the particular case $\mathcal{M}=\mathcal{T}$ and $j=m$. Since each colored tower has height 1 or at least 2 , we have $\left(\mathcal{T}^{s}, m, D_{n}\right)=\left(\left(\mathcal{T}_{1} \cup \mathcal{T}_{2}\right)^{s}, m, D_{n}\right)$. Let $\mathcal{U}^{2}(p)$ be the set of $U U$-steps of an elevated Dyck path $p \in D_{n}$. We will show

Lemma 2.1. $\left(\mathcal{T}_{1}^{s}, 1, D_{n}\right) \simeq\left(\left(\mathcal{U}^{2} \cup \mathcal{T}^{c}\right)^{s}, 1, D_{n-1}\right),\left(\mathcal{T}_{2}^{s}, 1, D_{n}\right) \simeq\left(\mathcal{T}^{s}, 1, D_{n-1}\right)$ and $\left(\mathcal{T}^{s}, m, D_{n}\right) \simeq\left(\mathcal{U}^{s}, m, D_{n-m}\right)$ for any $m, 1 \leq m \leq n$.

Proof. We use $U^{s}, U^{s} U^{s}$, and $t^{s}$ to represent a $U$-step, a $U U$-step, and a tower labeled by $s$. For a path $p \in D_{n}$ that has only one colored tower $t$ labeled by $s$, if the labeled and colored tower $t$ has height 1, i.e., $t \in \mathcal{T}_{1}(p)$, then $t$ is either located between two $U$-steps, i.e., $U t^{s} U \subseteq p$, or $t$ follows an uncolored tower $t_{1}$ from $\mathcal{T}^{c}(p)$, i.e., $t_{1} t^{s} \subseteq p$ where $t_{1} \in \mathcal{T}^{c}(p)$. Let $g_{1}:\left(\mathcal{T}_{1}^{s}, 1, D_{n}\right) \rightarrow\left(\left(\mathcal{U}^{2} \cup \mathcal{T}^{c}\right)^{s}, 1, D_{n-1}\right)$ be the map defined as follows. If $p=\cdots U t^{s} U \cdots \in\left(\mathcal{T}_{1}^{s}, 1, D_{n}\right)$, then $g_{1}(p)=$ $\cdots U^{s} U^{s} \cdots \in\left(\left(\mathcal{U}^{2}\right)^{s}, 1, D_{n-1}\right)$. If $p=\cdots t_{1} t^{s} \cdots \in\left(\mathcal{T}_{1}^{s}, 1, D_{n}\right)$, then $g_{1}(p)=$ $\cdots t_{1}^{s} \cdots \in\left(\left(\mathcal{T}^{c}\right)^{s}, 1, D_{n-1}\right)$. Notice that the tower $t_{1}$ is also an uncolored tower of the labeled path $g_{1}(p)$. This follows from the way we define the colored towers. It is obvious that the map $g_{1}$ is a bijection.

For a path $p \in D_{n}$ that has only one colored tower $t$ labeled by $s$, if the labeled and colored tower $t$ has height at least 2, i.e., $t \in \mathcal{T}_{2}(p)$, then $t=U t_{2} D$ where $t_{2}$ is a tower. Let $g_{2}:\left(\mathcal{T}_{2}^{s}, 1, D_{n}\right) \rightarrow\left(\mathcal{T}^{s}, 1, D_{n-1}\right)$ be the map defined as follows. If $p=\cdots t^{s} \cdots=\cdots\left(U t_{2} D\right)^{s} \cdots \in\left(\mathcal{T}_{2}^{s}, 1, D_{n}\right)$, then $g_{2}(p)=\cdots t_{2}^{s} \cdots \in\left(\mathcal{T}^{s}, 1, D_{n-1}\right)$. Notice that the tower $t_{2}$ is also a colored tower of the labeled path $g_{2}(p)$. This follows from the way we define the colored towers. It is clear that the map $g_{2}$ is a bijection.

We will next prove $\left(\mathcal{T}^{s}, m, D_{n}\right) \simeq\left(\left(\mathcal{U}^{2} \cup \mathcal{T} \cup \mathcal{T}^{c}\right)^{s}, m, D_{n-m}\right)$ for any $m$. The bijection for the case $m=1$ can be obtained by combining the bijections $g_{1}$ and $g_{2}$, namely, let $f_{1}:\left(\mathcal{T}^{s}, 1, D_{n}\right) \rightarrow\left(\left(\mathcal{U}^{2} \cup \mathcal{T}^{c} \cup \mathcal{T}\right)^{s}, 1, D_{n-1}\right)$ be the bijection defined as follows: $f_{1}(p)=g_{1}(p)$ if $p \in\left(\mathcal{T}_{1}^{s}, 1, D_{n}\right)$ and $f_{1}(p)=g_{2}(p)$ if $p \in\left(\mathcal{T}_{2}^{s}, 1, D_{n}\right)$. We can extend the bijection $f_{1}$ to the general bijection $f_{m}$ from the set $\left(\mathcal{T}^{s}, m, D_{n}\right)$ to the set $\left(\left(\mathcal{U}^{2} \cup \mathcal{T} \cup \mathcal{T}^{c}\right)^{s}, m, D_{n-m}\right)$ as follows. For any $p \in\left(\mathcal{T}^{s}, m, D_{n}\right)$, suppose $p$ has $t_{1}, t_{2}, \ldots, t_{m}$ colored towers labeled by $s$ from left to right. For every labeled tower $t_{i}$ that has height 1 and $U t_{i}^{s} U \subseteq p$, we label the $U$-steps next to $t_{i}$ by $s$ and remove $t_{i}$ from $p$, i.e., $U^{s} U^{s} \subseteq f_{m}(p)$. For every labeled tower $t_{i}$ that has height 1 and $t t_{i}^{s} \subseteq p$, we label the tower $t$ by $s$ and remove $t_{i}$ from $p$, i.e., $t^{s} \subseteq f_{m}(p)$. For every labeled tower $t_{i}$ that has height at least 2, i.e., $t_{i}^{s}=(U t D)^{s} \subseteq p$, we label $t$ by $s$ and remove a $U$-step and a $D$-step from the tower $t_{i}$, i.e., $t^{s} \subseteq f_{m}(p)$. In fact, the map $f_{m}$ on the path $p$ is equivalent to applying the bijection $f_{1}$ on 
each labeled tower $t_{i}$ of $p$ from left to right. It follows that $f_{m}$ is a bijection and therefore $\left(\mathcal{T}^{s}, m, D_{n}\right) \simeq\left(\left(\mathcal{U}^{2} \cup \mathcal{T} \cup \mathcal{T}^{c}\right)^{s}, m, D_{n-m}\right)$ holds for any $m$. It remains to prove $\mathcal{U}^{2}(p) \cup \mathcal{T}(p) \cup \mathcal{T}^{c}(p) \simeq \mathcal{U}(p)$ for any $p \in D_{n}$.

In view of $\mathcal{T}^{c}(p) \cup \mathcal{T}(p)=\mathcal{T}^{*}(p)$ for any $p \in D_{n}$, we next show for any $p \in D_{n}$, $\mathcal{U}^{2}(p) \cup \mathcal{T}^{*}(p) \simeq \mathcal{U}(p)$. Each $U$-step is either part of a $U U$-step or part of a peak $P$ by considering the step that follows this $U$-step. Each peak $P$ is contained in only one tower from $\mathcal{T}^{*}(p)$ and each tower from $\mathcal{T}^{*}(p)$ has only one peak. Therefore $\mathcal{U}(p) \simeq \mathcal{U}^{2}(p) \cup \mathcal{T}^{*}(p)$ for any $p \in D_{n}$ and the proof is complete.

For a path $p$, we name the first $U$-step of a tower $t \in \mathcal{T}_{2}(p)$ bottom. Let $\mathcal{B}(p)$ be the set of bottoms contained in a path $p$, and let $\left(\left(\mathcal{T}^{s}, \mathcal{B}^{w}\right),(m, r), D_{n}\right)$ be the set of elevated Dyck paths $p \in D_{n}$ having exactly $m$ colored towers labeled by $s$ and among these $m$ colored towers there are exactly $r$ bottoms labeled by $w$. Let $\left(\left(\mathcal{T}^{s}, \mathcal{T}^{w}\right),(m, r), D_{n}\right)$ be the set of elevated Dyck paths $p \in D_{n}$ having exactly $m$ colored towers labeled by $s$ and among these $m$ colored towers there are exactly $r$ colored towers labeled by $w$. We will prove

Lemma 2.2. $\left(\left(\mathcal{T}^{s}, \mathcal{B}^{w}\right),(m, r), D_{n}\right) \simeq\left(\left(\mathcal{T}^{s}, \mathcal{T}^{w}\right),(m, r), D_{n-r}\right)$.

Proof. For a path $p \in\left(\left(\mathcal{T}^{s}, \mathcal{B}^{w}\right),(m, r), D_{n}\right)$ and for every colored tower $t \in \mathcal{T}_{2}(p)$ labeled by $s$ whose bottom is labeled by $w$, we remove the bottom and a $D$ step from $t$, and label the remaining colored tower by $s$ and $w$. For a path $\bar{p} \in\left(\left(\mathcal{T}^{s}, \mathcal{T}^{w}\right),(m, r), D_{n-r}\right)$ and for every colored tower $\bar{t} \in \mathcal{T}(\bar{p})$ labeled by $s, w$, we replace the tower $\bar{t}^{s, w}$ by the tower $U^{w} \bar{t}^{s} D$. This yields a bijection.

Now we are in position to prove (1.6).

Proof. Let $F_{n}(q)=q N_{n}(q)$. Then (1.6) is equivalent to

$$
\frac{F_{n}(q)}{(1-q)^{n}}=\frac{q}{1-q}+\sum_{k=1}^{n-1} \frac{F_{n-k}(q)}{(1-q)^{n-k}} \sum_{m=0}^{k-1}(-1)^{m}\left(\begin{array}{c}
k-1 \\
m
\end{array}\right)\left(\begin{array}{c}
n-m \\
k
\end{array}\right) \frac{q}{(1-q)^{m+1}}
$$

where $\frac{F_{n}(q)}{(1-q)^{n}}=\sum_{k=1}^{n} \frac{N_{n, k} q^{k}}{(1-q)^{n}}$ counts the number of elevated Dyck paths of length $2 n+2$ where each $U$-step, except the first $U$-step, has weight $\frac{1}{1-q}$ and each peak $U D$ has weight $q$. Before we proceed to the combinatorial proof, we first transform $\frac{q}{(1-q)^{m+1}}$ according to our needs, namely,

$$
\begin{aligned}
& \sum_{m=0}^{k-1}(-1)^{m}\left(\begin{array}{c}
k-1 \\
m
\end{array}\right)\left(\begin{array}{c}
n-m \\
k
\end{array}\right) \frac{q}{(1-q)^{m+1}} \\
= & \sum_{i=0}^{k-1} \sum_{m \geq i}(-1)^{m}\left(\begin{array}{c}
k-1 \\
m
\end{array}\right)\left(\begin{array}{c}
n-m \\
k
\end{array}\right)\left(\begin{array}{c}
m \\
i
\end{array}\right)\left(\frac{q}{1-q}\right)^{i+1} \\
= & \sum_{m=1}^{k}(-1)^{m-1}\left(\begin{array}{c}
k-1 \\
m-1
\end{array}\right)\left(\begin{array}{c}
n-k+1 \\
m
\end{array}\right)\left(\frac{q}{1-q}\right)^{m} .
\end{aligned}
$$


Consequently, we can express (2.1) as

$$
\frac{F_{n}(q)}{(1-q)^{n}}=\frac{q}{1-q}+\sum_{m=1}^{n-1}(-1)^{m-1} \sum_{k=m}^{n-m+1} \frac{F_{n-k}(q)}{(1-q)^{n-k}}\left(\begin{array}{c}
k-1 \\
m-1
\end{array}\right)\left(\begin{array}{c}
n-k+1 \\
m
\end{array}\right)\left(\frac{q}{1-q}\right)^{m} .
$$

We observe that adding a peak $P$ right between double $U$-steps contributes weight $\frac{q}{1-q}$, while adding a peak $P$ right between $U D$-steps contributes weight $\frac{1}{1-q}$. In contrast to the proof of (1.1), the reduction from $\frac{F_{n}(q)}{(1-q)^{n}}$ to $\frac{F_{n-1}(q)}{(1-q)^{n-1}}$ by removing a peak highly depends on the type of the $U$-step we choose.

Next we will derive an identity for the generating function of weighted elevated Dyck paths. This can also be obtained by directly applying the inclusion-exclusion principle. We will show this identity in both ways. We set $g_{n, k}(\mathcal{T})=\mid\left\{p \in D_{n}\right.$ : $|\mathcal{T}(p)|=k\} \mid$ and define $g_{n, k}(\mathcal{T}, q)$ to be the generating function of $g_{n, k}(\mathcal{T})$ that weights each $U$-step, except the first $U$-step, of an elevated Dyck path $p \in D_{n}$ by $\frac{1}{1-q}$ and each peak $U D$ of an elevated Dyck path $p \in D_{n}$ by $q$. Recall that we denote by $\left(\mathcal{M}^{s}, m, D_{n}\right)$ the set of elevated Dyck paths $p \in D_{n}$ having exactly $m$ elements from the set $\mathcal{M}(p)$ labeled by $s$, and that $a_{n, m}(\mathcal{M})=\left|\left(\mathcal{M}^{s}, m, D_{n}\right)\right|$. Furthermore, we denote by $\left(\left(\bigcup_{i=1}^{k} \mathcal{M}_{i}\right)^{s}, m, D_{n}\right)$ the set of elevated Dyck paths $p \in D_{n}$ having exactly $m$ elements from the set $\bigcup_{i=1}^{k} \mathcal{M}_{i}(p)$ labeled by $s$, and define $a_{n, m}\left(\bigcup_{i=1}^{k} \mathcal{M}_{i}\right)=\left|\left(\left(\bigcup_{i=1}^{k} \mathcal{M}_{i}\right)^{s}, m, D_{n}\right)\right|$. Then, analogous to the proof of (1.1), we consider $\mathcal{M}=\mathcal{T}$. Let $a_{n, m}(\mathcal{T}, q)$ be the generating function of $a_{n, m}(\mathcal{T})$ where each $U$-step, except the first $U$-step, of an elevated Dyck path $p \in D_{n}$ is weighted by $\frac{1}{1-q}$ and each peak $P$ of an elevated Dyck path $p \in D_{n}$ is weighted by q. Then

$$
a_{n, m}(\mathcal{T})=\sum_{k \geq m}\left(\begin{array}{c}
k \\
m
\end{array}\right) g_{n, k}(\mathcal{T}), \quad a_{n, m}(\mathcal{T}, q)=\sum_{k \geq m}\left(\begin{array}{c}
k \\
m
\end{array}\right) g_{n, k}(\mathcal{T}, q) .
$$

In particular for $m=0$, we have $a_{n, 0}(\mathcal{T})=C_{n}$ and $a_{n, 0}(\mathcal{T}, q)=\frac{F_{n}(q)}{(1-q)^{n}}$. By introducing the generating function $G_{n}(x, q)$ of $g_{n, k}(\mathcal{T}, q)$, we obtain

$$
G_{n}(x, q)=\sum_{k \geq 1} g_{n, k}(\mathcal{T}, q) x^{k}=\sum_{m=0}^{n} \frac{G_{n}^{(m)}(1, q)}{m !}(x-1)^{m}=\sum_{m=0}^{n} a_{n, m}(\mathcal{T}, q)(x-1)^{m},
$$

where $G_{n}^{(m)}(1, q)=\left.\frac{\partial^{m} G_{n}(x, q)}{\partial x^{m}}\right|_{x=1}$. By setting $x=0$, we get

$$
a_{n, 0}(\mathcal{T}, q)=\frac{F_{n}(q)}{(1-q)^{n}}=\sum_{m=1}^{n}(-1)^{m-1} a_{n, m}(\mathcal{T}, q) .
$$

In fact (2.3) can also be derived by directly applying the inclusion-exclusion method. To be precise, we adopt the notations from [12. Let $A_{i}$ be the set of elevated Dyck paths in $D_{n}$ whose $i$-th tower (in the left-to-right order) is a colored tower. Then $A_{1}, \ldots, A_{n}$ are all the subsets of the set $D_{n}$ and $A_{1} \cup \cdots \cup A_{n}=D_{n}$. For each subset $T$ of $[n]$, let $A_{T}=\bigcap_{i \in T} A_{i}$ with $A_{\varnothing}=D_{n}$. Here $A_{T}$ is the set of elevated Dyck paths in $D_{n}$ whose $i$-th tower (in the left-to-right order) is a colored tower for every $i \in T$. For $0 \leq m \leq n$, we set $S_{m}=\sum_{|T|=m}\left|A_{T}\right|$ where the sum runs over all the $m$-subsets $T$ of $[n]$. Therefore $S_{m}$ equals the number of elevated Dyck paths in $D_{n}$ that have $m$ colored towers labeled by $s$, which is $a_{n, m}(\mathcal{T})$. So according to the 
principle of inclusion-exclusion, the number $\left|\bar{A}_{1} \cap \cdots \cap \bar{A}_{n}\right|$ of elevated Dyck paths in $D_{n}$ that have no colored towers equals

$$
\left|\bar{A}_{1} \cap \cdots \cap \bar{A}_{n}\right|=\sum_{m=0}^{n}(-1)^{m} S_{m}=\sum_{m=0}^{n}(-1)^{m} a_{n, m}(\mathcal{T}) .
$$

Since every elevated Dyck path has at least one colored tower, we have $\mid \bar{A}_{1} \cap \cdots \cap$ $\bar{A}_{n} \mid=g_{n, 0}(\mathcal{T})=0$. Consequently, $a_{n, 0}(\mathcal{T})=\sum_{m=1}^{n}(-1)^{m-1} a_{n, m}(\mathcal{T})$. In terms of the weighted elevated Dyck paths, (2.3) follows.

In order to use (2.3) to prove (2.2), we will need to give an explicit expression for $a_{n, m}(\mathcal{T}, q)$. For any path $p \in D_{n}$ and any two colored towers $t_{1}, t_{2} \in \mathcal{T}(p), t_{1}$ and $t_{2}$ are disjoint. We start by counting $a_{n, 1}(\mathcal{T}, q)$, which is the generating function for the elevated Dyck paths $p \in D_{n}$ having one colored tower labeled by $s$. Equivalently, $\left|\left(\mathcal{T}^{s}, 1, D_{n}\right)\right|=a_{n, 1}(\mathcal{T})$. In view of Lemma 2.1, we get $a_{n, 1}(\mathcal{T})=a_{n-1,1}(\mathcal{U})$ and

$$
\begin{aligned}
a_{n, 1}(\mathcal{T}, q) & =\frac{q}{1-q} a_{n-1,1}\left(\mathcal{U}^{2} \cup \mathcal{T}^{c}, q\right)+\frac{1}{1-q} a_{n-1,1}(\mathcal{T}, q) \\
& =\frac{q}{1-q} a_{n-1,1}(\mathcal{U}, q)+a_{n-1,1}(\mathcal{T}, q)=\sum_{k=1}^{n-1} \frac{q}{1-q} a_{n-k, 1}(\mathcal{U}, q)+a_{1,1}(\mathcal{T}, q) \\
(2.4) \quad & =\sum_{k=1}^{n-1}(n-k+1) \frac{F_{n-k}(q)}{(1-q)^{n-k}} \frac{q}{1-q}+\frac{q}{1-q} .
\end{aligned}
$$

Next we will count $a_{n, m}(\mathcal{T}, q)$ for $m \geq 2$. For a path $p$, recall that the first $U$-step of a tower $t \in \mathcal{T}_{2}(p)$ is named bottom. For a given path $p \in D_{n}$ with $m$ colored towers labeled by $s$, let $\mathcal{T}_{2, m}$ denote the subset of these $m$ towers that have height $\geq 2$. Since each $U$-step has weight $\frac{1}{1-q}$ and each tower in $\mathcal{T}_{2, m}$ has one bottom, the weight on the bottoms of path $p \in D_{n}$ is $\left(\frac{1}{1-q}\right)^{\left|\mathcal{T}_{2, m}\right|}$, which is equal to

$(2.5)\left(\frac{1}{1-q}\right)^{\left|\mathcal{T}_{2, m}\right|}=\left(\frac{q}{1-q}\right)^{\left|\mathcal{T}_{2, m}\right|}+\sum_{r=1}^{\left|\mathcal{T}_{2, m}\right|}(-1)^{r-1}\left(\begin{array}{c}\left|\mathcal{T}_{2, m}\right| \\ r\end{array}\right)\left(\frac{1}{1-q}\right)^{\left|\mathcal{T}_{2, m}\right|-r}$.

We will separate the weight $\left(\frac{1}{1-q}\right)^{\left|\mathcal{T}_{2, m}\right|}$ on the bottoms of path $p$ according to (2.5). The term $\left(\frac{q}{1-q}\right)^{\left|\mathcal{T}_{2, m}\right|}$ on the right-hand side of (2.5) corresponds to the case that each bottom is weighted by $\frac{q}{1-q}$. Accordingly, we set $f_{n, m, 0}(\mathcal{T}, q)$ to be the generating function of elevated Dyck paths $p \in D_{n}$ that have $m$ colored towers labeled by $s$ where each bottom has weight $\frac{q}{1-q}$, each $U$-step, other than the first $U$-step and the bottom, has weight $\frac{1}{1-q}$ and each peak has weight $q$. In the same way as for the counting of $a_{n, 1}(\mathcal{T}, q)$, the set $\left(\mathcal{T}^{s}, m, D_{n}\right)$ is in one-to-one correspondence to the set $\left(\mathcal{U}^{s}, m, D_{n-m}\right)$ as shown in Lemma 2.1. Therefore,

$$
\begin{aligned}
a_{n, m}(\mathcal{T}) & =a_{n-m, m}(\mathcal{U}), \\
f_{n, m, 0}(\mathcal{T}, q) & =\left(\frac{q}{1-q}\right)^{m} a_{n-m, m}(\mathcal{U}, q) .
\end{aligned}
$$

The term $\left(\begin{array}{c}\left|\mathcal{T}_{2, m}\right| \\ r\end{array}\right)\left(\frac{1}{1-q}\right)^{\left|\mathcal{T}_{2, m}\right|-r}$ on the right-hand side of (2.5) represents the number of ways to choose $r$ towers from the set $\mathcal{T}_{2, m}$ where each bottom of these $r$ towers has weight 1 and each bottom of the remaining $\left(\left|\mathcal{T}_{2, m}\right|-r\right)$ towers has weight $\frac{1}{1-q}$. Accordingly, for $r \geq 1$ we define $f_{n, m, r}(\mathcal{T})$ to be the number of elevated Dyck paths $p \in D_{n}$ that have $m$ colored towers labeled by $s$ and among these $m$ colored towers 
there are exactly $r$ bottoms labeled by $w$, i.e., $\left|\left(\left(\mathcal{T}^{s}, \mathcal{B}^{w}\right),(m, r), D_{n}\right)\right|=f_{n, m, r}(\mathcal{T})$. Furthermore, let $f_{n, m, r}(\mathcal{T}, q)$ be the generating function of $f_{n, m, r}(\mathcal{T})$ where each bottom labeled by $w$ has weight 1 , each $U$-step, other than the bottom labeled by $w$ and the first $U$-step, has weight $\frac{1}{1-q}$ and each peak has weight $q$. From Lemma 2.2 . we obtain $f_{n, m, r}(\mathcal{T})=\left|\left(\left(\mathcal{T}^{s}, \mathcal{T}^{w}\right),(m, r), D_{n-r}\right)\right|$ and therefore

$$
f_{n, m, r}(\mathcal{T})=\left(\begin{array}{c}
m \\
r
\end{array}\right) a_{n-r, m}(\mathcal{T}), \quad f_{n, m, r}(\mathcal{T}, q)=\left(\begin{array}{c}
m \\
r
\end{array}\right) a_{n-r, m}(\mathcal{T}, q)
$$

By multiplying both sides of (2.5) by the weights on the $U$-steps (except the first $U$-step and the bottoms) and the weights on the peaks of a path $p \in D_{n}$, and summing over all the paths in $D_{n}$, we get

$$
a_{n, m}(\mathcal{T}, q)=f_{n, m, 0}(\mathcal{T}, q)+\sum_{r=1}^{m}(-1)^{r-1} f_{n, m, r}(\mathcal{T}, q)
$$

Together with (2.6) and (2.7), we have

$$
\begin{aligned}
a_{n, m}(\mathcal{T}, q) & =\left(\frac{q}{1-q}\right)^{m} a_{n-m, m}(\mathcal{U}, q)+\sum_{r=1}^{m}(-1)^{r-1}\left(\begin{array}{c}
m \\
r
\end{array}\right) a_{n-r, m}(\mathcal{T}, q) \\
(2.8) & =\left(\frac{q}{1-q}\right)^{m}\left(\begin{array}{c}
n-m+1 \\
m
\end{array}\right) \frac{F_{n-m}(q)}{(1-q)^{n-m}}+\sum_{r=1}^{m}(-1)^{r-1}\left(\begin{array}{c}
m \\
r
\end{array}\right) a_{n-r, m}(\mathcal{T}, q) .
\end{aligned}
$$

We will employ generating functions to solve (2.8). Let $A_{m}(x)$ be the generating function for $a_{n, m}(\mathcal{T}, q)$ and $m \geq 2$, i.e., the $n$-th coefficient of $A_{m}(x)$-denoted by $\left[x^{n}\right] A_{m}(x)$-is $a_{n, m}(\mathcal{T}, q)$. By multiplying both sides of (2.8) by $x^{n}$ and summing over all $n$, we obtain

$$
\begin{aligned}
A_{m}(x) & =(1-x)^{-m} \sum_{n \geq m}\left(\begin{array}{c}
n-m+1 \\
m
\end{array}\right)\left(\frac{q}{1-q}\right)^{m} \frac{F_{n-m}(q)}{(1-q)^{n-m}} x^{n}, \\
a_{n, m}(\mathcal{T}, q) & =\left[x^{n}\right] A_{m}(x)=\sum_{i \geq 0}\left(\begin{array}{c}
n-m-i+1 \\
m
\end{array}\right)\left(\frac{q}{1-q}\right)^{m}\left(\begin{array}{c}
m+i-1 \\
m-1
\end{array}\right) \frac{F_{n-m-i}(q)}{(1-q)^{n-m-i}} \\
& =\sum_{k=m}^{n-m+1}\left(\begin{array}{c}
n-k+1 \\
m
\end{array}\right)\left(\begin{array}{c}
k-1 \\
m-1
\end{array}\right) \frac{F_{n-k}(q)}{(1-q)^{n-k}}\left(\frac{q}{1-q}\right)^{m} .
\end{aligned}
$$

In combination of (2.3) and (2.4), the proof of (2.2) is complete.

\section{Generalize (1.6) to Ballot numbers}

The Ballot numbers $B_{n, r}$ are defined by $B_{n, r}=\frac{r+1}{2 n+r+1}\left(\begin{array}{c}2 n+r+1 \\ n\end{array}\right)$. They count the number of paths $p$ from $(0,0)$ to $(2 n+r, r)$ with allowed $U$-step and $D$-step and each path $p$ can be decomposed as $p_{1} U p_{2} \cdots U p_{r+1}$ with $p_{i}, 1 \leq i \leq r+1$, a Dyck path. Let $\frac{M_{n, r}(q)}{(1-q)^{n}}$ be the generating function for the path $p_{1} p_{2} \cdots p_{r+1}$ that has total length $2 n$ where each peak of $p_{1} p_{2} \cdots p_{r+1}$ has weight $q$ and each $U$-step of $p_{1} p_{2} \cdots p_{r+1}$ has weight $\frac{1}{1-q}$. Then we can generalize (1.6) to an equation for $M_{n, r}(q)$, i.e.,

$$
\frac{M_{n, r}(q)}{(1-q)^{n}}=\frac{q(r+1)}{1-q}+\sum_{m=1}^{n-1}(-1)^{m-1} \sum_{k \geq m} \frac{q^{m} M_{n-k, r}(q)}{(1-q)^{n-k+m}}\left(\begin{array}{c}
k-1 \\
m-1
\end{array}\right)\left(\begin{array}{c}
n-k+1+r \\
m
\end{array}\right) .
$$


The proof follows similarly to that for (1.6) and is omitted here. Next we will prove (1.7) by involution and the inclusion-exclusion method on the partitions and answer Andrews' questions on the property of $T_{r}(n, q)$ given in (1.8).

\section{Properties of $T_{r}(n, q)$}

We say a polynomial $f(x)=a_{n} x^{n}+\cdots+a_{0}$ is reciprocal if $f(x)=x^{n} f\left(\frac{1}{x}\right)$, i.e., $a_{r}=a_{n-r}$. A sequence $a_{0}, a_{1}, \ldots, a_{n}$ of real numbers is said to be unimodal if for some $0 \leq j \leq n$ we have $a_{0} \leq \cdots \leq a_{j-1} \leq a_{j} \geq a_{j+1} \geq \cdots \geq a_{n}$. We say a polynomial $f(x)=a_{n} x^{n}+\cdots+a_{1} x+a_{0}$ is unimodal if the sequence $a_{0}, a_{1}, \ldots, a_{n}$ is unimodal. If $f(x)$ and $g(x)$ are unimodal and reciprocal polynomials with nonnegative coefficients, then $f(x) g(x)$ is also unimodal and reciprocal. Here we say $f(x)$ is a positive polynomial if all the coefficients of $f(x)$ are nonnegative. We say the polynomial $f(x)$ has nonnegative coefficients (resp. nonpositive coefficients) up to $x^{r}$ if for any $0 \leq i \leq r$, the coefficient of $x^{i}$ in the polynomial $f(x)$ is nonnegative (resp. nonpositive). $\omega \in \mathbb{C}$ is a primitive $k$-th root of unity if and only if $\omega^{k}=1$ and for any $d \in \mathbb{Z}^{+}$and $d<k, \omega^{d} \neq 1$. The $k$-th cyclotomic polynomial $\Phi_{k}(x) \in \mathbb{Z}[x]$ is the polynomial whose roots are the primitive $k$-th roots of unity. Let $\omega$ be any primitive $k$-th root of unity, and the polynomial $f(x)$ satisfies $f(\omega)=0$. Then $\Phi_{k}(x) \mid f(x)$. The $q$-Lucas theorem is the following:

Proposition 1 ( $q$-Lucas theorem). Let $m, k, d$ be positive integers, and write $m=$ $a d+b$ and $k=r d+s$, where $0 \leq b, s \leq d-1$. Let $\omega$ be any primitive $d$-th root of unity. Then

$$
\left[\begin{array}{c}
m \\
k
\end{array}\right]_{\omega}=\left(\begin{array}{l}
a \\
r
\end{array}\right)\left[\begin{array}{l}
b \\
s
\end{array}\right]_{\omega} .
$$

Theorem 4.1. $T_{r}(n, q)$ is a polynomial in $q$. If $n$ is even, $T_{r}(n, q)$ is a positive polynomial. If $n$ is odd, $(1+q) T_{r}(n, q)$ is a positive polynomial. In case $n=2 r-1$, $T_{r}(2 r-1,-q)$ is a positive polynomial.

Proof. We can simplify $T_{r}(n, q)$ defined in (1.8) into

$$
\begin{aligned}
T_{r}(n, q) & =q^{r^{2}-r} \frac{1}{[n]_{q}}\left[\begin{array}{c}
n \\
r
\end{array}\right]_{q^{2}}\left[\begin{array}{c}
2 n-2 r \\
n-1
\end{array}\right]_{q} \\
& =q^{r^{2}-r}\left[\begin{array}{c}
n-1 \\
r
\end{array}\right]_{q^{2}} \frac{\left(1+q^{n}\right)}{[n-1]_{q}}\left[\begin{array}{c}
2 n-2 r-1 \\
n-2
\end{array}\right]_{q} .
\end{aligned}
$$

Consequently from (4.1) and (4.2) we have for $r \geq 1$,

$$
\begin{aligned}
T_{r}(n, q) & =\left([n]_{q}-q[n-1]_{q}\right) T_{r}(n, q) \\
& =q^{r^{2}-r}\left[\begin{array}{l}
n \\
r
\end{array}\right]_{q^{2}}\left[\begin{array}{c}
2 n-2 r \\
n-1
\end{array}\right]_{q}-q^{r^{2}-r}\left[\begin{array}{c}
n-1 \\
r
\end{array}\right]_{q^{2}}\left(q+q^{n+1}\right)\left[\begin{array}{c}
2 n-2 r-1 \\
n-2
\end{array}\right]_{q},
\end{aligned}
$$

which implies $T_{r}(n, q)$ is a polynomial.

We will next show the polynomial $T_{r}(n, q)$ is positive for even $n$ and the polynomial $(1+q) T_{r}(n, q)$ is positive for odd $n$. Let $d=\operatorname{gcd}(n, r)$; then

$$
\begin{aligned}
T_{r}(n, q) & =q^{r^{2}-r}\left[\begin{array}{l}
n \\
r
\end{array}\right]_{q^{2}} \frac{[d]_{q^{2}}}{[n]_{q^{2}}} \frac{[n]_{q^{2}}}{[d]_{q^{2}}[2 n-2 r+1]_{q}}\left[\begin{array}{c}
2 n-2 r+1 \\
n
\end{array}\right]_{q} \\
& =q^{r^{2}-r}\left[\begin{array}{l}
n \\
r
\end{array}\right]_{q^{2}} \frac{[d]_{q^{2}}}{[n]_{q^{2}}} \frac{\left(1+q^{n}\right)(1-q)}{1-q^{2 d}}\left[\begin{array}{c}
2 n-2 r \\
n-1
\end{array}\right]_{q} .
\end{aligned}
$$


It has been proved by Brunetti et al. that $\left[\begin{array}{l}n \\ r\end{array}\right]_{q^{2}} \frac{[d]_{q^{2}}}{[n]_{q^{2}}}$ is a positive polynomial based on the fact that polynomial $[d]_{q}\left[\begin{array}{l}n \\ r\end{array}\right]_{q}$ is unimodal and reciprocal [4]. In the same way, let $m=\operatorname{gcd}(n, 2 r-1)$; then $\frac{[m]_{q}}{[2 n-2 r+1]_{q}}\left[\begin{array}{c}2 n-2 r+1 \\ n\end{array}\right]_{q}$ is a positive polynomial. It remains to prove $\frac{\left(1+q^{n}\right)(1-q)}{1-q^{2 d}}\left[\begin{array}{c}2 n-2 r \\ n-1\end{array}\right]_{q}$ is a positive polynomial for even $n$ and $\frac{\left(1+q^{n}\right)\left(1-q^{2}\right)}{1-q^{2 d}}\left[\begin{array}{c}2 n-2 r \\ n-1\end{array}\right]_{q}$ is a positive polynomial for odd $n$. Recall that $m=\operatorname{gcd}(n, 2 r-1)$. First we observe $m$ must be odd and therefore

$$
\frac{\left(1+q^{n}\right)(1-q)}{1-q^{2 d}}\left[\begin{array}{c}
2 n-2 r \\
n-1
\end{array}\right]_{q}=\frac{[m]_{q}[m]_{-q}}{[2 n-2 r+1]_{q}}\left[\begin{array}{c}
2 n-2 r+1 \\
n
\end{array}\right]_{q} \frac{[n]_{q^{2}}}{[m]_{q^{2}}[d]_{q^{2}}} .
$$

Here $\frac{[n]_{q}}{[m]_{q}[d]_{q}}$ is a polynomial since $\operatorname{gcd}(m, d)=1$ and $m|n, d| n$. Together with the fact that $\frac{[m]_{q}}{[2 n-2 r+1]_{q}}\left[\begin{array}{c}2 n-2 r+1 \\ n\end{array}\right]_{q}$ is a polynomial, it follows that $\frac{\left(1+q^{n}\right)(1-q)}{1-q^{2 d}}\left[\begin{array}{c}2 n-2 r \\ n-1\end{array}\right]_{q}$ is a polynomial. We next prove $\frac{\left(1+q^{n}\right)(1-q)}{1-q^{2 d}}\left[\begin{array}{c}2 n-2 r \\ n-1\end{array}\right]_{q}$ is a positive polynomial if $n$ is even. If $n$ is even, then $\frac{1-q}{1-q^{2 d}}\left[\begin{array}{c}2 n-2 r \\ n-1\end{array}\right]_{q}$ is a polynomial since for any $x \mid(2 d)$ and $x>1, x \mid(2 n-2 r)$ and $x \nmid(n-1)$. If not, $x \mid(n-1)$, and then $x \mid \operatorname{gcd}(n-1,2)=1$, contradicting the assumption. Thus by using the $q$-Lucas theorem, $\frac{1-q}{1-q^{2 d}}\left[\begin{array}{c}2 n-2 r \\ n-1\end{array}\right]_{q}$ is a polynomial. To be precise, for any $x \in \mathbb{Z}^{+}$such that $x \mid(2 d)$ and $x>1$, suppose $2 n-2 r=c_{1} x, n-1=c_{2} x+t_{1}$ for some $t_{1} \neq 0$, and let $\rho$ be any primitive $x$-th root of unity. Then by applying the $q$-Lucas theorem, we have $\left[\begin{array}{c}2 n-2 r \\ n-1\end{array}\right]_{\rho}=\left[\begin{array}{c}c_{1} x \\ c_{2} x+t_{1}\end{array}\right]_{\rho}=$ $\left(\begin{array}{l}c_{1} \\ c_{2}\end{array}\right)\left[\begin{array}{l}0 \\ t_{1}\end{array}\right]_{\rho}=0$. This shows for any $x \mid(2 d)$ and $x>1, \Phi_{x}(q) \mid\left[\begin{array}{c}2 n-2 r \\ n-1\end{array}\right]_{q}$. Since $[2 d]_{q}=$ $\prod_{x \mid(2 d)} \Phi_{x}(q)$ and any two cyclotomic polynomials are relatively prime, we can conclude that $\prod_{x>1}^{x \mid(2 d)} \Phi_{x}(q) \mid\left[\begin{array}{c}2 n-2 r \\ n-1\end{array}\right]_{q}$ and therefore $\frac{1-q}{1-q^{2 d}}\left[\begin{array}{c}2 n-2 r \\ n-1\end{array}\right]_{q}=\frac{1}{[2 d]_{q}}\left[\begin{array}{c}2 n-2 r \\ n-1\end{array}\right]_{q}$ is a polynomial. In view of the unimodality of polynomial $\left[\begin{array}{c}2 n-2 r \\ n-1\end{array}\right]_{q},(1-q)\left[\begin{array}{c}2 n-2 r \\ n-1\end{array}\right]_{q}$ has nonnegative coefficients up to $q^{\left\lfloor\frac{(n-1)(n-2 r+1)}{2}\right\rfloor}$. Therefore we expand $\frac{1}{1-q^{2 d}}$ as a power series at $q=0$ and obtain

$$
\frac{(1-q)}{1-q^{2 d}}\left[\begin{array}{c}
2 n-2 r \\
n-1
\end{array}\right]_{q}=(1-q)\left[\begin{array}{c}
2 n-2 r \\
n-1
\end{array}\right]_{q}+q^{2 d}(1-q)\left[\begin{array}{c}
2 n-2 r \\
n-1
\end{array}\right]_{q}+\cdots,
$$

which implies that the polynomial $\frac{(1-q)}{1-q^{2 d}}\left[\begin{array}{c}2 n-2 r \\ n-1\end{array}\right]_{q}$ has nonnegative coefficients up to $q^{\left\lfloor\frac{(n-1)(n-2 r+1)}{2}\right\rfloor}$. Together with the reciprocity of $\frac{(1-q)}{1-q^{2 d}}\left[\begin{array}{c}2 n-2 r \\ n-1\end{array}\right]_{q}$, we conclude that $\frac{(1-q)}{1-q^{2 d}}\left[\begin{array}{c}2 n-2 r \\ n-1\end{array}\right]_{q}$ has nonnegative coefficients and therefore $T_{r}(n, q)$ is a positive polynomial if $n$ is even.

In the same way, we can prove that $\frac{\left(1-q^{2}\right)}{1-q^{2 d}}\left[\begin{array}{c}2 n-2 r \\ n-1\end{array}\right]_{q}$ is a positive polynomial if $n$ is odd. This implies $(1+q) T_{r}(n, q)$ is a positive polynomial if $n$ is odd.

By setting $q \rightarrow-q$ and $n \rightarrow 2 r-1$, we can obtain

$$
T_{r}(2 r-1,-q)=q^{r^{2}-r}\left[\begin{array}{c}
2 r-1 \\
r
\end{array}\right]_{q^{2}} \frac{1+q}{1+q^{2 r-1}}=q^{r^{2}-r}[2 r-1]_{q} C_{r-1}\left(q^{2}\right),
$$

which indicates $T_{r}(2 r-1,-q)$ has nonnegative coefficients. 


\section{Proof of (1.7)}

We transform $T_{r}(n, q)$ from (4.3) into

$$
\begin{aligned}
T_{r}(n, q) & =q^{r^{2}-r}\left[\begin{array}{l}
n-1 \\
r-1
\end{array}\right]_{q^{2}}\left[\begin{array}{c}
2 n-2 r+1 \\
n
\end{array}\right]_{q}-q^{r^{2}-r}\left[\begin{array}{c}
n-1 \\
r
\end{array}\right]_{q^{2}} q\left[\begin{array}{c}
2 n-2 r-1 \\
n-2
\end{array}\right]_{q} \\
& +q^{r^{2}+r}\left[\begin{array}{c}
n-1 \\
r
\end{array}\right]_{q^{2}}\left[\begin{array}{c}
2 n-2 r-1 \\
n
\end{array}\right]_{q} \text { valid for } n \geq 2 r+1, \text { and } \\
T_{r}(n, q) & =q^{r^{2}-r}\left[\begin{array}{l}
n-1 \\
r-1
\end{array}\right]_{q^{2}}\left[\begin{array}{c}
2 n-2 r+1 \\
n
\end{array}\right]_{q}-q^{r^{2}-r}\left[\begin{array}{c}
n-1 \\
r
\end{array}\right]_{q^{2}} q\left[\begin{array}{c}
2 n-2 r-1 \\
n-2
\end{array}\right]_{q}
\end{aligned}
$$

valid for $2 r-1 \leq n \leq 2 r$.

For simplicity, we set $a_{n}^{(r)}=q^{r^{2}+r}\left[\begin{array}{c}n-1 \\ r\end{array}\right]_{q^{2}}\left[\begin{array}{c}2 n-2 r-1 \\ n\end{array}\right]_{q}$. Then we can write $T_{r}(n, q)=$ $a_{n}^{(r-1)}+a_{n}^{(r)}-q^{r^{2}-r}\left[\begin{array}{c}n-1 \\ r\end{array}\right]_{q^{2}} q\left[\begin{array}{c}2 n-2 r-1 \\ n-2\end{array}\right]_{q}$ for $n \geq 2 r+1$ and $T_{r}(n, q)=a_{n}^{(r-1)}-$ $q^{r^{2}-r}\left[\begin{array}{c}n-1 \\ r\end{array}\right]_{q^{2}} q\left[\begin{array}{c}2 n-2 r-1 \\ n-2\end{array}\right]_{q}$ for $2 r-1 \leq n \leq 2 r$. First we observe

$$
\begin{aligned}
& \sum_{r=1}^{\left\lfloor\frac{n+1}{2}\right\rfloor-1}(-1)^{r-1}\left(a_{n}^{(r-1)}+a_{n}^{(r)}\right)+(-1)^{\left\lfloor\frac{n+1}{2}\right\rfloor-1} a_{n}^{\left(\left\lfloor\frac{n+1}{2}\right\rfloor-1\right)} \\
= & a_{n}^{(0)}=\left[\begin{array}{c}
2 n-1 \\
n
\end{array}\right]_{q}=C_{n}(q)+q\left[\begin{array}{c}
2 n-1 \\
n-2
\end{array}\right]_{q}
\end{aligned}
$$

and it remains to prove

$$
\left[\begin{array}{c}
2 n-1 \\
n-2
\end{array}\right]_{q}=\sum_{r=1}^{\left\lfloor\frac{n+1}{2}\right\rfloor}(-1)^{r-1} q^{r^{2}-r}\left[\begin{array}{c}
n-1 \\
r
\end{array}\right]_{q^{2}}\left[\begin{array}{c}
2 n-2 r-1 \\
n-2
\end{array}\right]_{q} .
$$

We interpret (5.2) in terms of partitions. A partition $\lambda$ is defined as a finite sequence of nonnegative integers $\left(\lambda_{1}, \lambda_{2}, \cdots, \lambda_{m}\right)$ in the weakly decreasing order $\lambda_{1} \geq \lambda_{2} \geq$ $\cdots \geq \lambda_{m}$. Each $\lambda_{i} \neq 0$ is called a part of $\lambda$. The number and the sum of parts of $\lambda$ are denoted by $\ell(\lambda)$ and $|\lambda|$, respectively. The partition-theoretic interpretation of the $q$-binomial $\left[\begin{array}{c}n+k \\ k\end{array}\right]_{q}$ is $\left[\begin{array}{c}n+k \\ k\end{array}\right]_{q}=\sum_{\substack{\ell(\lambda) \leq k \\ \lambda_{1} \leq n}} q^{|\lambda|}$ as given in [1. Therefore we have

$$
q^{n-2 r+1}\left[\begin{array}{c}
2 n-2 r-1 \\
n-2
\end{array}\right]_{q}=\sum_{\substack{\ell(\nu)=n-2 r+1 \\
\nu_{1} \leq n-1}} q^{|\nu|}, \quad q^{r^{2}+r}\left[\begin{array}{c}
n-1 \\
r
\end{array}\right]_{q^{2}}=\sum_{\substack{\mu_{1}>\mu_{2}>\cdots>\mu_{r} \geq 1 \\
\mu_{i} \leq n-i}} q^{2|\mu|} .
$$

Given two partitions $\mu$ and $\nu$, say $\mu=\left(\mu_{1}, \ldots, \mu_{m}\right)$ and $\nu=\left(\nu_{1}, \ldots, \nu_{n}\right)$, let $\mu^{2} \cup \nu$ be the partition whose parts are $\mu_{1}, \mu_{1}, \ldots, \mu_{m}, \mu_{m}, \nu_{1}, \ldots, \nu_{n}$ in decreasing order. Let $\mu \cup \nu$ be the partition whose parts are $\mu_{1}, \ldots, \mu_{m}, \nu_{1}, \ldots, \nu_{n}$ in decreasing order, and let $\nu \backslash\left(\mu_{1}, \ldots, \mu_{m}\right)$ be the partition obtained from $\nu$ by removing the parts equal to $\mu_{1}, \ldots, \mu_{m}$.

For any pair $(\mu, \nu)$ such that $n-1 \geq \mu_{1}>\cdots>\mu_{r} \geq 1$ where $\mu_{i} \leq n-i$ and $n-1 \geq \nu_{1} \geq \cdots \geq \nu_{n-2 r+1} \geq 1$, let $x$ be the smallest part in the partition $\mu^{2} \cup \nu$ with repetition. We shall construct a new pair $\left(\mu^{\prime}, \nu^{\prime}\right)$ as follows:

(1) If $\mu_{r}=x$, then we choose $\mu^{\prime}=\left(\mu_{1}, \cdots, \mu_{r-1}\right)$ and $\nu^{\prime}=\nu \cup(x, x)$.

(2) Otherwise, $\nu_{n-2 r}=\nu_{n-2 r+1}=x$, and we choose $\mu^{\prime}=\left(\mu_{1}, \cdots, \mu_{r}, x\right)$ and $\nu^{\prime}=\nu \backslash(x, x)$. 
Consequently $2|\mu|+|\nu|=2\left|\mu^{\prime}\right|+\left|\nu^{\prime}\right|$ but the lengths of $\mu$ and $\mu^{\prime}$ differ by 1 . Indeed the map $f:(\mu, \nu) \mapsto\left(\mu^{\prime}, \nu^{\prime}\right)$ is an involution, since for any pair $\left(\mu^{\prime}, \nu^{\prime}\right)$, we can define the inverse map $g:\left(\mu^{\prime}, \nu^{\prime}\right) \mapsto(\mu, \nu)$ as follows. Let $y$ be the smallest part in the partition $\mu^{\prime 2} \cup \nu^{\prime}$ with repetition. If $y \notin \mu^{\prime}$, then we choose $\mu=\mu^{\prime} \cup(y)$ and $\nu=\nu^{\prime} \backslash(y, y)$. Otherwise we choose $\mu=\mu^{\prime} \backslash(y)$ and $\nu=\nu^{\prime} \cup(y, y)$. Clearly, $g$ is the inverse of $f$. This involution leads to (5.2).

In exactly the same way as for the proof of (1.1) and (1.6), we can prove (5.2) by counting the partitions that have exactly $k$ different parts with repetition labeled by $s$. Let $g_{n, k}\left(q^{n+1}\left[\begin{array}{c}2 n-1 \\ n-2\end{array}\right]_{q}\right)$ count the partitions $\lambda$ with $\lambda_{1} \leq n-1$ and $\ell(\lambda)=n+1$ such that there are exactly $k$ different parts with repetition. Then the number of partitions $\lambda$ with $\lambda_{1} \leq n-1$ and $\ell(\lambda)=n+1$, such that there are exactly $r$ different parts with repetition labeled by $s$, is

$$
\sum_{k \geq r}\left(\begin{array}{l}
k \\
r
\end{array}\right) g_{n, k}\left(q^{n+1}\left[\begin{array}{c}
2 n-1 \\
n-2
\end{array}\right]_{q}\right)=q^{r^{2}+r}\left[\begin{array}{c}
n-1 \\
r
\end{array}\right]_{q^{2}} q^{n-2 r+1}\left[\begin{array}{c}
2 n-2 r-1 \\
n-2
\end{array}\right]_{q} .
$$

Again, by employing the generating function of $g_{n, k}\left(q^{n+1}\left[\begin{array}{c}2 n-1 \\ n-2\end{array}\right]_{q}\right)$, we have

$$
\begin{aligned}
G_{n}(x, q) & =\sum_{k \geq 0} g_{n, k}\left(q^{n+1}\left[\begin{array}{c}
2 n-1 \\
n-2
\end{array}\right]_{q}\right) x^{k}=\sum_{r \geq 0} \frac{G_{n}^{(r)}(1, q)}{r !}(x-1)^{r} \\
& =\sum_{r \geq 0} q^{r^{2}+r}\left[\begin{array}{c}
n-1 \\
r
\end{array}\right]_{q^{2}} q^{n-2 r+1}\left[\begin{array}{c}
2 n-2 r-1 \\
n-2
\end{array}\right]_{q}(x-1)^{r} .
\end{aligned}
$$

By setting $x=0$ on both sides, we get (5.2) and therefore (1.7) follows. From the proof we see that the expression of $T_{r}(n, q)$ given in (5.1) implies that the sieving process works on the partitions counted by $\left[\begin{array}{c}2 n-1 \\ n-2\end{array}\right]_{q}$.

\section{Generalize (1.7) to $q$-Ballot numbers}

The $q$-Ballot numbers $B_{j}(n, q)$ are defined by

$$
B_{j}(n, q)=\frac{[j]_{q}}{[2 n+j]_{q}}\left[\begin{array}{c}
2 n+j \\
n
\end{array}\right]_{q}=\frac{[j]_{q}}{[n]_{q}}\left[\begin{array}{c}
2 n+j-1 \\
n-1
\end{array}\right]_{q} .
$$

They count the major index of lattice paths from $(0,0)$ to $(2 n+j-1,-j+1)$ with allowed $U$-step and $D$-step that never go below $y=-j+1$; see [9]. In particular, $B_{1}(n, q)=C_{n}(q)$ and $B_{r}(n, 1)=B_{n, r-1}$. Furthermore,

$$
B_{j}(n, q)=\left[\begin{array}{c}
2 n+j-2 \\
n
\end{array}\right]_{q}-q^{j}\left[\begin{array}{c}
2 n+j-2 \\
n-2
\end{array}\right]_{q}
$$

also counts the partitions $\lambda$ with $\lambda_{1} \leq n+j-2$ and $\ell(\lambda) \leq n$ whose successive ranks are all $<j-1$; see [2]. We generalize (1.7) to an equation for $q$-Ballot numbers as follows.

Theorem 6.1. The q-Ballot numbers $B_{j}(n, q)$ satisfy

$$
\begin{aligned}
B_{j}(n, q) & =\sum_{r=1}^{n}(-1)^{r-1} T_{r}^{(j)}(n, q), \\
T_{r}^{(j)}(n, q) & =q^{r^{2}-r}\left[\begin{array}{l}
n \\
r
\end{array}\right]_{q^{2}}\left[\begin{array}{c}
2 n+j-1-2 r \\
n-1
\end{array}\right]_{q} \frac{[j]_{q}}{[n]_{q}},
\end{aligned}
$$


where $T_{r}^{(j)}(n, q)$ is a polynomial for $n \geq 2 r-j$. In particular $T_{r}^{(j)}(2 r-j,-q)$ is a positive polynomial if $j \leq r$.

Proof. First we shall show $T_{r}^{(j)}(n, q)$ is a polynomial for $n \geq 2 r-j$. By setting $d=\operatorname{gcd}(n, r)$ and $m=\operatorname{gcd}(n, 2 r-j)$ we express

$$
\begin{aligned}
T_{r}^{(j)}(n, q) & =q^{r^{2}-r}\left[\begin{array}{l}
n \\
r
\end{array}\right]_{q^{2}} \frac{[d]_{q^{2}}}{[n]_{q^{2}}}\left[\begin{array}{c}
2 n+j-1-2 r]_{q} \\
n-1
\end{array}\right]_{q}[n]_{q^{2}}[j]_{q} \\
& =q^{r^{2}-r}\left[\begin{array}{l}
n \\
r
\end{array}\right]_{q^{2}} \frac{[d]_{q^{2}}}{[n]_{q^{2}}} \frac{[n]_{q^{2}}[j]_{q}}{[d]_{q^{2}}[m]_{q}} \frac{[m]_{q}}{[2 n+j-2 r]_{q}}\left[\begin{array}{c}
2 n+j-2 r]_{q} \\
n
\end{array}\right]_{q},
\end{aligned}
$$

where $\left[\begin{array}{c}n \\ r\end{array}\right]_{q^{2}}\left[\frac{[d]_{q^{2}}}{[n]_{q^{2}}}\right.$ and $\frac{[m]_{q}}{[2 n+j-2 r]_{q}}\left[\begin{array}{c}2 n+j-2 r \\ n\end{array}\right]_{q}$ are positive polynomials as proved in Theorem 4.1 and it remains to show $\frac{[n]_{q^{2}}[j]_{q}}{[d]_{q^{2}}[\mathrm{~m}]_{q}}$ is a polynomial. First we can simplify

$$
\frac{[n]_{q^{2}}[j]_{q}}{[d]_{q^{2}}[m]_{q}}=\frac{[2 n]_{q}[j]_{q}}{[2 d]_{q}[m]_{q}}
$$

For any $m_{1} \mid m$ and $m_{1}>1$, if $m_{1} \mid j$, then $\Phi_{m_{1}}(q) \mid[j]_{q}$ and therefore $\Phi_{m_{1}}(q) \mid$ $\frac{[2 n]_{q}}{[2 d]_{q}}[j]_{q}$. Otherwise, $m_{1} \nmid j$ and therefore $m_{1} \nmid d$. In fact, $m_{1} \nmid(2 d)$. If not, then $m_{1} \mid(2 r)$. Since $m_{1} \mid m$, hence $m_{1} \mid(2 r-j)$. This leads to $m_{1} \mid j$, contradicting the assumption. Let $\omega$ be any $m_{1}$-th primitive root of unity, i.e., $\omega^{m_{1}}=1$. Then we have $[2 n]_{\omega}=0$ and $[2 d]_{\omega} \neq 0$ which implies $\Phi_{m_{1}}(q) \mid \frac{[2 n]_{q}}{[2 d]_{q}}$. It follows that $\Phi_{m_{1}}(q) \mid \frac{[2 n]_{q}}{[2 d]_{q}}[j]_{q}$ for any $m_{1} \mid m$ and $m_{1}>1$. Since any two cyclotomic polynomials are relatively prime, we have that $\frac{[2 n]_{q}[j]_{q}}{[2 d]_{q}[m]_{q}}$ is a polynomial. Therefore

$$
\frac{[n]_{q^{2}}[j]_{q}}{[d]_{q^{2}}[m]_{q}} \frac{[m]_{q}}{[2 n+j-2 r]_{q}}\left[\begin{array}{c}
2 n+j-2 r \\
n
\end{array}\right]_{q}=\frac{\left(1+q^{n}\right)(1-q)}{\left(1-q^{2 d}\right)}[j]_{q}\left[\begin{array}{c}
2 n+j-2 r-1 \\
n-1
\end{array}\right]_{q}
$$

and $T_{r}^{(j)}(n, q)$ are polynomials.

In particular, $T_{r}^{(j)}(2 r-j, q)$ is a polynomial. We next prove $T_{r}^{(j)}(2 r-j,-q)$ is a positive polynomial if $j \leq r$. If $j$ is even, then

$$
T_{r}^{(j)}(2 r-j,-q)=q^{r^{2}-r}\left[\begin{array}{c}
2 r-j \\
r
\end{array}\right]_{q^{2}} \frac{1-q^{j}}{1-q^{2 r-j}}
$$

holds. Here $q^{r^{2}-r}\left[\begin{array}{c}2 r-j \\ r\end{array}\right]_{q^{2}}\left(1-q^{j}\right)$ has nonnegative coefficients up to $q^{\left\lfloor\frac{3 r^{2}-r-2 r j}{2}\right\rfloor}$ since $q^{\frac{r^{2}-r}{2}}\left[\begin{array}{c}2 r-j \\ r\end{array}\right]_{q}$ is unimodal and $j$ is even. In combination with the expansion of $\frac{1}{1-q^{2 r-j}}$ at $q=0$ where $j \leq r$, we conclude that $T_{r}^{(j)}(2 r-j,-q)$ is a positive polynomial for even $j$. However, if $j$ is odd, we cannot prove the claim in the same way as before. If $j$ is odd, we have

$$
T_{r}^{(j)}(2 r-j,-q)=q^{r^{2}-r}\left[\begin{array}{c}
2 r-j \\
r
\end{array}\right]_{q^{2}} \frac{1+q^{j}}{1+q^{2 r-j}} .
$$


Since $q^{\frac{r^{2}-r}{2}}\left[\begin{array}{c}2 r-j \\ r\end{array}\right]_{q}$ is a positive polynomial and since $j$ is odd, we find that $\left(1+q^{j}\right) q^{r^{2}-r}\left[\begin{array}{c}2 r-j \\ r\end{array}\right]_{q^{2}}$ is a positive polynomial. But in view of the expansion of $\frac{1}{1+q^{2 r-j}}$ at $q=0$, we get

$$
T_{r}^{(j)}(2 r-j,-q)=\left(1+q^{j}\right) q^{r^{2}-r}\left[\begin{array}{c}
2 r-j \\
r
\end{array}\right]_{q^{2}}-q^{2 r-j}\left(1+q^{j}\right) q^{r^{2}-r}\left[\begin{array}{c}
2 r-j \\
r
\end{array}\right]_{q^{2}}+\cdots,
$$

where the polynomial $-q^{2 r-j}\left(1+q^{j}\right) q^{r^{2}-r}\left[\begin{array}{c}2 r-j \\ r\end{array}\right]_{q^{2}}$ could make the first half of the coefficients of $\left(1+q^{j}\right) q^{r^{2}-r}\left[\begin{array}{c}2 r-j \\ r\end{array}\right]_{q^{2}}$ become negative. Therefore, we choose to prove $T_{r}^{(j)}(2 r-j,-q)$ in (6.4) to be a positive polynomial by considering

$$
q T_{r}^{(j)}(2 r-j, q)=q^{r^{2}-r+1}\left[\begin{array}{c}
2 r-j \\
r
\end{array}\right]_{q^{2}} \frac{1-q^{j}}{1-q^{2 r-j}} .
$$

The claim that polynomial $T_{r}^{(j)}(2 r-j,-q)$ has nonnegative coefficients is equivalent to the claim that polynomial $q T_{r}^{(j)}(2 r-j, q)$ has nonnegative coefficients for the odd powers of $q$, and nonpositive coefficients for the even powers of $q$. Now for $q T_{r}^{(j)}(2 r-j, q)$ the unimodality of $q^{\frac{r^{2}-r}{2}}\left[\begin{array}{c}2 r-j \\ r\end{array}\right]_{q}$ implies that $q^{r^{2}-r+1}\left[\begin{array}{c}2 r-j \\ r\end{array}\right]_{q^{2}}\left(1-q^{j}\right)$ has nonnegative coefficients for the odd powers of $q$ up to $q^{\left\lfloor\frac{3 r^{2}-r-2 r j+1}{2}\right\rfloor}$. By expanding $\frac{1}{1-q^{2 r-j}}$ as a power series at $q=0$, we have that the odd powers of $q$ in the polynomial $q T_{r}^{(j)}(2 r-j, q)$, come from

$$
\left(1+q^{4 r-2 j}+q^{8 r-4 j}+\cdots\right)\left(1-q^{2 r}\right) q^{r^{2}-r+1}\left[\begin{array}{c}
2 r-j \\
r
\end{array}\right]_{q^{2}},
$$

from which we can conclude that the coefficents of the odd powers of $q$, up to $q^{\left\lfloor\frac{3 r^{2}-r-2 r j+1}{2}\right\rfloor}$ in the polynomial $q T_{r}^{(j)}(2 r-j, q)$ are nonnegative. Furthermore, we observe that the maximal degree $d_{\max }$ of $q$ in the polynomial $q T_{r}^{(j)}(2 r-j, q)$ is $3 r^{2}-3 r-2 r j+2 j+1$ and the minimal degree $d_{\min }$ of $q$ in the polynomial $q T_{r}^{(j)}(2 r-j, q)$ is $r^{2}-r+1$. Let $a_{i}$ be the coefficient of $q^{i}$ in the polynomial $q T_{r}^{(j)}(2 r-j, q)$. Then from the reciprocity of the polynomial $q T_{r}^{(j)}(2 r-j, q)$ we get $a_{i}=a_{j}$ if $j+i=d_{\text {max }}+d_{\text {min }}=4 r^{2}-2 r j-4 r+2 j+2$. This implies for $i$ odd and $a_{i} \geq 0, j$ is odd and $a_{j} \geq 0$. Therefore we can conclude that the coefficients of all the odd powers of $q$ in the polynomial $q T_{r}^{(j)}(2 r-j, q)$ are nonnegative. This is equivalent to say that the coefficients of all the even powers of $q$ in the polynomial $T_{r}^{(j)}(2 r-j, q)$ are nonnegative. By expanding $\frac{1-q^{j}}{1-q^{2 r-j}}$ at $q=0$, we have that the even powers of $q$ in the polynomial $q T_{r}^{(j)}(2 r-j, q)$ come from

$$
-q^{j}\left(1+q^{4 r-2 j}+q^{8 r-4 j}+\cdots\right)\left(1-q^{2 r-2 j}\right) q^{r^{2}-r+1}\left[\begin{array}{c}
2 r-j \\
r
\end{array}\right]_{q^{2}},
$$

where $-q^{r^{2}-r+1+j}\left[\begin{array}{c}2 r-j \\ r\end{array}\right]_{q^{2}}\left(1-q^{2 r-2 j}\right)$ is a polynomial that has nonpositive coefficients for the even powers of $q$, up to $q^{\left.\frac{\left\lfloor r^{2}-2 r j-r+j+1\right.}{2}\right\rfloor}$. Again from the reciprocity of polynomial $q T_{r}^{(j)}(2 r-j, q)$, we conclude that the coefficients of all the even powers of $q$ in the polynomial $q T_{r}^{(j)}(2 r-j, q)$ are nonpositive. That is to say, the coefficients of all the odd powers of $q$ in the polynomial $T_{r}^{(j)}(2 r-j,-q)$ are nonnegative. Now it remains to prove (6.1). By similar techniques as used to prove (1.7), we 
consider the partitions counted by $q^{n+j}\left[\begin{array}{c}2 n+j-1 \\ n-1\end{array}\right]_{q}$ and let $g_{n, k}\left(q^{n+j}\left[\begin{array}{c}2 n+j-1 \\ n-1\end{array}\right]_{q}\right)$ count the partitions $\lambda$ with $\lambda_{1} \leq n$ and $\ell(\lambda)=n+j$ such that there are exactly $k$ different parts with repetition. By following the same techniques used in the proof of (1.7) of Section 5 ( see the proof of (5.4)), we can derive the identity

$$
q^{n+j}\left[\begin{array}{c}
2 n+j-1 \\
n-1
\end{array}\right]_{q}=\sum_{r=1}^{n}(-1)^{r-1} q^{r^{2}+r}\left[\begin{array}{l}
n \\
r
\end{array}\right]_{q^{2}} q^{n+j-2 r}\left[\begin{array}{c}
2 n+j-2 r-1 \\
n-1
\end{array}\right]_{q},
$$

which is equivalent to 6.1).

Conjecture. Here we conjecture that if $n$ is odd, then for $m \geq n \geq 1$ the polynomial $\left(1+q^{n}\right)\left[\begin{array}{c}m \\ n-1\end{array}\right]_{q}$ is unimodal. If $n$ is even, then for any even $j \neq 0$ and $m \geq n \geq 1$, the polynomial $\left(1+q^{n}\right)[j]_{q}\left[\begin{array}{c}m \\ n-1\end{array}\right]_{q}$ is unimodal. This, in combination with (6.3), implies that $T_{r}(n, q)$ is a positive polynomial for odd $n$ and $n \geq 2 r+1$, and $T_{r}^{(j)}(n, q)$ given in (6.2) is a positive polynomial for $n \geq 2 r-j+1$.

\section{ACKNOWLEDGEMENT}

The authors would like to thank the anonymous reviewers for their helpful comments that greatly helped to improve the final version of this manuscript.

\section{REFERENCES}

[1] George E. Andrews, The theory of partitions, Cambridge Mathematical Library, Cambridge University Press, Cambridge, 1998. Reprint of the 1976 original. MR.1634067 (99c:11126)

[2] George E. Andrews, On the difference of successive Gaussian polynomials, J. Statist. Plann. Inference 34 (1993), no. 1, 19-22, DOI 10.1016/0378-3758(93)90030-A. MR1209986 (94f:11010)

[3] George E. Andrews, q-Catalan identities, The legacy of Alladi Ramakrishnan in the mathematical sciences, Springer, New York, 2010, pp. 183-190, DOI 10.1007/978-1-4419-6263-8_10. MR:2744261 (2012f:05018)

[4] S. Brunetti, A. Del Lungo, and F. Del Ristoro, An equipartition property for the distribution of multiset permutation inversions, Adv. in Appl. Math. 27 (2001), no. 1, 41-50, DOI 10.1006/aama.2001.0725. MR.1835676 (2002c:05011)

[5] J. Cigler, Operatormethoden für q-Identitäten. V. q-Catalan-Bäume (German), Österreich. Akad. Wiss. Math.-Natur. Kl. Sitzungsber. II 205 (1996), 175-182 (1997). MR.1601338 (98m:05013)

[6] J. Cigler, Some remarks about $q$-Chebyshev polynomials and $q$-Catalan numbers and related results, arXiv:1312.2767.

[7] George Gasper and Mizan Rahman, Basic hypergeometric series, 2nd ed., Encyclopedia of Mathematics and its Applications, vol. 96, Cambridge University Press, Cambridge, 2004. With a foreword by Richard Askey. MR2128719 (2006d:33028)

[8] Thomas Koshy, Catalan numbers with applications, Oxford University Press, Oxford, 2009. MR2526440(2010g:05008)

[9] Christian Krattenthaler, Counting lattice paths with a linear boundary. II. q-ballot and qCatalan numbers (English, with German summary), Österreich. Akad. Wiss. Math.-Natur. Kl. Sitzungsber. II 198 (1989), no. 4-7, 171-199. MR1064033 (92c:05020)

[10] Michel Lassalle, Narayana polynomials and Hall-Littlewood symmetric functions, Adv. in Appl. Math. 49 (2012), no. 3-5, 239-262, DOI 10.1016/j.aam.2012.06.002. MR3017958

[11] Vladimir N. Sachkov, Probabilistic methods in combinatorial analysis, Encyclopedia of Mathematics and its Applications, vol. 56, Cambridge University Press, Cambridge, 1997. Translated from the Russian; Revised by the author. MR1453118 (98g:60022)

[12] Richard P. Stanley, Enumerative combinatorics. Vol. 2, Cambridge Studies in Advanced Mathematics, vol. 62, Cambridge University Press, Cambridge, 1999. With a foreword by Gian-Carlo Rota and appendix 1 by Sergey Fomin. MR.1676282 (2000k:05026) 
[13] Herbert S. Wilf, generatingfunctionology, 2nd ed., Academic Press, Inc., Boston, MA, 1994. MR $1277813(95 \mathrm{a}: 05002)$

[14] Doron Zeilberger, The method of creative telescoping, J. Symbolic Comput. 11 (1991), no. 3, 195-204, DOI 10.1016/S0747-7171(08)80044-2. MR1103727(92c:33005)

Department of Computer Science, University of Kaiserslautern, Kaiserslautern, Germany

E-mail address: jin@cs.uni-kl.de

Department of Computer Science, University of Kaiserslautern, Kaiserslautern, Germany - And - Department of Mathematics and Computer Science, University of Southern Denmark, Denmark

E-mail address: nebel@cs.uni-kl.de 